\title{
ON COX-ROSS-RUBINSTEIN PRICING FORMULA FOR PRICING COMPOUND OPTION
}

\author{
JAVED HUSSAIN*, BAREERAH KHAN \\ Sukkur IBA University, Pakistan \\ ${ }^{*}$ Corresponding author: javed.brohi@iba-suk.edu.pk
}

\begin{abstract}
The fundamental objective of this paper is twofold. Firstly, to derive the Cox-Ross-Rubinstein type new formula for risk neutral pricing of European compound call option, where the underlying asset is also a European call option. Thirdly, to prove that our newly derived CRR risk neutral pricing formula for compound call option, converges in distribution to the well known, continuous time Black-Scholes formula for pricing the compound call option on call.
\end{abstract}

\section{INTRODUCTION}

A compound option is an option that has further an option as the underlying asset. Compound options were first studied by Geske (1979, [9]), using a partial differential equation method and Fourier integrals. Afterward, several other approaches were introduced for pricing methods for compound options. For instance, Lajeri-Chaherli $(2002$, [15]) used the martingale method and by computing the expectation of truncated bivariate normal variables, priced the compound options. Agliardi (2003, [1]) priced a generalized timedependent compound calls. Gukhal (2004, [12]) proposed a model for valuation of the compound option, in which the underlying option follows the log-normal jump-diffusion process. Fouque and Han (2005, [13]) employed a perturbation techniques to compute the prices of compound options. Chiarella and Kang (2011, [5]) and $(2014,[4])$ evaluated American style compound option with stochastic volatility. Griebsch (2013, [11])

Received 2019-10-25; accepted 2019-11-19; published 2020-01-02.

2010 Mathematics Subject Classification. 91G20.

Key words and phrases. binomial pricing; compound options; Cox-Ross-Rubinstein framework; convergence in distribution. (C)2020 Authors retain the copyrights of their papers, and all open access articles are distributed under the terms of the Creative Commons Attribution License. 
used FFT (Fast Fourier Transform) to price the European style compound option prices, where volatility assumed to be stochastic.

Since most studies have been done through continuous time approach so in this paper we emphasize on pricing the compound option through the discrete time Cox-Ross-Rubinstein (CRR) approach, we refer the reader to chapter 6 of [7]. They key novelty of the work is the detailed proof that discrete time CRR price of the compound option converges to the continuous time pricing formula of the compound option. We now give a brief description of all sections of this paper.

Section 1 is running introduction. The section 2 gives A short introduction to mechanics of the compound option and its continuous time formula. Section 3 has been devoted to deriving, in a greater detail, the CRR formula for the risk-neutral pricing the European-style compound option on European call option. In Section 4, we have given the proof that the CRR price of compound option converges to the well-known continuous time pricing formula, in distribution. options. section 6 comprises of conclusion.

\section{CRR Formula for Pricing Compound Options}

This section comprises of a brief introduction to compound option and our new result on the derivation of its CRR premium of European call option when the underlying asset is a European call option on stock.

2.1. Introduction to Compound Option. Here we briefly illustrate a compound option which is a particular type of exotic option. To do that we need a brief time line here.

$$
T_{0-----} T_{1------} T_{2}
$$

Today is time zero, $T_{1}$ is the point time in future and $T_{2}$ will be even later than that. If today at $T=0$ we purchase a call option we are buying a right to exercise a price of $K_{1}$ and exchange for that we will receive a call option. So in this case we are purchasing a call option on a call option. Mainly, there can be four possible variations of compound options.

1. Call with underlying Call.

2. Call with underlying Put.

3. Put with underlying Call.

4. Put with underlying Put.

So today at $T=0$ when we purchase a compound option it gives us a right to going forward to exercise that compound option. So let's just say that stock increases little bit here at $T=1$ and we exercise compound option. We pay the strike price of $K_{1}$ and get the option not the stock. So we only do that if the value at $T=1$ of call option is greater than the strike price $C>K_{1}$. So after having exercise the compound, we now in fact own the more familiar plain vanilla call option on stock and we do need another strike price $K_{2}$. The 
compound option has two strike prices, first only to purchase a compound option which is $K_{1}$ the price we pay in order to exercise initially to purchase a call option and that call option has $K_{2}$ its own strike price. If we go forward in time, say stock move in our favor such that at $T_{1}$, there is an intrinsic value in that call option and if $S>K_{2}$ then we exercise the underlying option by paying the strike price $K_{2}$ and receiving the underlying asset (in this case it's a stock).

When assumption of geometric Brownian motion is made, European style compound option can analytically be valued in terms of integral of bivariate normal distribution. The value of European call on call option at $T=0$ is

$$
C=S N\left(a_{1}, b_{1} ; \sqrt{\frac{T_{1}}{T_{2}}}\right)-e^{-r T_{2}} K_{2} N\left(a_{2}, b_{2} ; \sqrt{\frac{T_{1}}{T_{2}}}\right)-e^{-r T_{1}} K_{1} N\left(a_{2}\right)
$$

where

$$
\begin{aligned}
& a_{1}=\frac{\ln \left(\frac{S_{0}}{S^{*}}\right)+\left(r-\frac{\sigma^{2}}{2}\right) T_{1}}{\sigma \sqrt{T_{1}}} ; a_{2}=a_{1}+\sigma \sqrt{T_{1}} \\
& b_{1}=\frac{\ln \left(\frac{S_{0}}{K_{2}}\right)+\left(r-\frac{\sigma^{2}}{2}\right) T_{2}}{\sigma \sqrt{T_{2}}} ; b_{2}=b_{1}+\sigma \sqrt{T_{2}}
\end{aligned}
$$

$N=$ cumulative bivariate normal distribution function.

$S^{*}=$ critical stock price at $T_{1}$ such that compound option is in the money. We refer to [14] for further details.

\section{Derivation of CRR Compound Option Formula}

Now we move towards the key objective of the paper i.e. pricing the compound option through binomial approach. We aim to construct a CRR formula for the compound options. Let me state the key theorem of the section.

Theorem 3.1. In a viable Cox-Ross-Rubinstein model with parameters $S_{0}, T, r, u$ and $d$ the fair price at time 0 of a European compound call option with expiry $m$ and strike $K_{1}$, contingent upon a European call option with expiry $n$ and strike $K_{2}$, can be given as:

$$
C=S_{0} B\left(a, b ; p^{\prime} ; n, n-m\right)-(1+r)^{-(n-m)} K_{2} B\left(a, b ; p^{\prime}, p ; n, n-m\right)
$$

$$
-(1+r)^{-m} K_{1} \psi(a ; m, p)
$$

where $p=\frac{r-u}{u-d}, p^{\prime}=\frac{p u}{(1+r)}, a=\left[\frac{\ln \frac{S^{*}}{S_{0}}-m \ln d}{\ln \left(\frac{u}{d}\right)}\right]+1$ and $b=\left[\frac{\ln \frac{K_{2}}{S_{0}}-n \ln d}{\ln \left(\frac{u}{d}\right)}\right]+1$, and $B(\cdot, \cdot ; \cdot)$ is the complementary distribution function of bi-variate Binomial distribution. 
Proof. Consider the discrete time line,

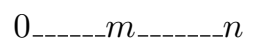

where $m, n \in \mathbb{N}$. We aim to price a compound call option where underlying asset is also a call option. The compound option will be exercised at $m$ (i.e. after $m$ steps/periods) and the underlying call option (whose value depends on underlying stock prices), will be exercised at $n$ steps/periods. By assuming the absence of arbitrage opportunities in the binomial model, the price of the underlying call option either moves up by a factor $u$ or down by a factor $d$ in each period. The probability of an upward movement is $p$ and the probability of a downward movement is $1-p$.

In order to compute the fair premium of the compound option at inception, we will begin by employing Theorem 5.47 of [7]. The payoff of the compound option can be given as,

$$
\text { payoff }=\max \left\{C_{s t d}\left(S_{m}, K_{2}, n-m\right)-K_{1}, 0\right\}
$$

where $\mathbb{E}$ is the risk neutral expectation, $K_{1}$ is the strike price for the compound option, and $C_{s t d}$ is the BlackScholes price of underlying call option at the maturity of compound option i.e. $m$. Thus by the Theorem 5.46 of [7], the premium of the compound option can be given as,

$$
C=(1+r)^{-m} \mathbb{E}\left(\max \left\{C_{s t d}\left(S_{m}, K_{2}, n-m\right)-K_{1}, 0\right\}\right)
$$

where $C_{s t d}\left(S_{m}, K_{2}, n-m\right)$ is the price of the underlying option. The expectation can be written more explicitly as,

$$
C=(1+r)^{-m} \sum_{i=0}^{m} \max \left(C_{s t d}\left(S_{m} u^{i} d^{m-i}, K_{2}, n-m\right)-K_{1}, 0\right)\left(\begin{array}{c}
m \\
i
\end{array}\right) p^{i}(1-p)^{m-i} .
$$

The compound option will be exercised i.e. its payoff will be nonzero if

$$
S_{m}>S^{*}
$$

where $S^{*}$ denotes the worth of the stock such that the underlying option is in the money at time $m$ i.e. $S^{*}$ solves the equation

$$
C_{\text {std }}\left(S_{m}, K_{2}, n-m\right)=K_{1}
$$

Assume that $a$ is the smallest time period $i$ such that.

$$
S_{m}>S^{*}
$$

Let us try to determine bound on $a$. As $S_{0} u^{a} d^{m-a}>S^{*}$, it follows that,

$$
a>\frac{\ln \frac{S^{*}}{S_{0}}-m \ln d}{\ln \left(\frac{u}{d}\right)} .
$$


Let us return back to equation (3.2) and write the expectation more explicitly.

$$
\begin{aligned}
C= & (1+r)^{-m} \sum_{i=a}^{m}\left(C_{\text {std }}\left(S_{m} u^{i} d^{m-i}, K_{2}, n-m\right) u^{i} d^{m-i}-K_{1}\right)\left(\begin{array}{c}
m \\
i
\end{array}\right) p^{i}(1-p)^{m-i} \\
= & (1+r)^{-m} \sum_{i=a}^{m} C_{\text {std }}\left(S_{m} u^{i} d^{m-i}, K_{2}, n-m\right)\left(\begin{array}{c}
m \\
i
\end{array}\right) p^{i}(1-p)^{m-i} u^{i} d^{m-i} \\
& -(1+r)^{-m} \sum_{i=a}^{m} K_{1}\left(\begin{array}{c}
m \\
i
\end{array}\right) p^{i}(1-p)^{m-i} \\
C= & (1+r)^{-m} \sum_{i=a}^{m} C_{\text {std }}\left(S_{m} u^{i} d^{m-i}, K_{2}, n-m\right)\left(\begin{array}{c}
m \\
i
\end{array}\right) p^{i}(1-p)^{m-i} u^{i} d^{m-i} \\
& -(1+r)^{-m} K_{1} \psi(a, m, p)
\end{aligned}
$$

where $\psi$ is the complementary Binomial distribution function,

$$
\psi(a, m, p)=\sum_{i=a}^{m}\left(\begin{array}{c}
m \\
i
\end{array}\right) p^{i}(1-p)^{m-i} .
$$

We want to further explore the above first term of equation (3.3). Here $C_{s t d}\left(S_{m}, K_{2}, n-m\right)$ represents the price of an underlying European option and the price is given as:

$$
\begin{gathered}
C_{s t d}\left(S_{m} u^{i} d^{m-i}, K_{2}, n-m\right)=(1+r)^{-(n-m)} \mathbb{E}\left(\left\{\max \left(S_{m}-K_{2}, 0\right)\right\}\right) \\
C_{s t d}\left(S_{m} u^{i} d^{m-i}, K_{2}, n-m\right)=(1+r)^{-(n-m)} \sum_{j=m}^{n} \max \left(\left(S_{m} u^{i} d^{m-i}\right) u^{j} d^{n-j}-K_{2}, 0\right) \times \\
\left(\begin{array}{c}
n-m \\
j
\end{array}\right) p^{j}(1-p)^{n-m-j} .
\end{gathered}
$$

Assume that $b<n$ in first instant such that

$$
b=\min \left\{a: S_{m} u^{j} d^{n-j}\right\}>K_{2} .
$$

Let us try to find bound on $b$. By definition $S_{m} u^{j} d^{n-j}>K_{2}$, therefore it follows that

$$
b>\frac{\ln \frac{K_{2}}{S_{m}}-n \ln d}{\ln \left(\frac{u}{d}\right)} .
$$

Now

$$
\begin{aligned}
C_{s t d}\left(S_{m} u^{i} d^{m-i}, K_{2}, n-m\right)= & (1+r)^{-(n-m)} \sum_{j=b}^{n} S_{m}\left(\begin{array}{c}
n-m \\
j
\end{array}\right) p^{j}(1-p)^{n-m-j} u^{j} d^{n-m-j} \\
& -(1+r)^{-(n-m)} K_{2} \sum_{j=b}^{n}\left(\begin{array}{c}
n-m \\
j
\end{array}\right) p^{j}(1-p)^{n-m-j} .
\end{aligned}
$$


Substitute the above equation in equation (3.3), we infer that,

$$
\begin{aligned}
& C=(1+r)^{-m} \sum_{i=a}^{m}\left(\begin{array}{c}
(1+r)^{-(n-m)} \sum_{j=b}^{n} S_{m}\left(\begin{array}{c}
n-m \\
j
\end{array}\right) p^{j}(1-p)^{n-m-j} u^{j} d^{n-m-j} \\
-(1+r)^{-(n-m)} K_{2} \sum_{j=b}^{n}\left(\begin{array}{c}
n-m \\
j
\end{array}\right) p^{j}(1-p)^{n-m-j}
\end{array}\right) \\
& \left(\begin{array}{c}
m \\
i
\end{array}\right) p^{i}(1-p)^{m-i} u^{i} d^{m-i}-(1+r)^{-m} K_{1} \psi(a, m, p) \text {. } \\
& C=(1+r)^{-n} \sum_{i=a}^{m} \sum_{j=b}^{n} S_{m}\left(\begin{array}{c}
m \\
i
\end{array}\right)\left(\begin{array}{c}
n-m \\
j
\end{array}\right) p^{i+j}(1-p)^{n-(i+j)} u^{i+j} d^{n-(i+j)} \\
& -(1+r)^{-n} K_{2} \sum_{i=a}^{m} \sum_{j=b}^{n}\left(\begin{array}{c}
m \\
i
\end{array}\right)\left(\begin{array}{c}
n-m \\
j
\end{array}\right) p^{i+j}(1-p)^{n-(i+j)} u^{i} d^{m-i} \\
& -K_{1} \psi\left(a, m, p^{\prime}\right) \\
& =\sum_{i=a}^{m} \sum_{j=b}^{n} S_{0}\left(\begin{array}{c}
m \\
i
\end{array}\right)\left(\begin{array}{c}
n-m \\
j
\end{array}\right)\left(\frac{p u}{(1+r)}\right)^{i+j}\left(\frac{(1-p) d}{(1+r)}\right)^{n-(i+j)} \\
& -(1+r)^{-(n-m)} K_{2} \sum_{i=a}^{m} \sum_{j=b}^{n}\left(\begin{array}{c}
m \\
i
\end{array}\right)\left(\begin{array}{c}
n-m \\
j
\end{array}\right)\left(\frac{p u}{(1+r)^{-(n-m)}}\right)^{i} \\
& \left(\frac{(1-p) d}{(1+r)^{-(n-m)}}\right)^{m-i} p^{j}(1-p)^{n-j}-(1+r)^{-(n-m)} K_{1} \psi(a, m, p),
\end{aligned}
$$

where $\left(\begin{array}{c}m \\ i\end{array}\right)=\frac{m !}{i !(m-i) !}$ and $\left(\begin{array}{c}n-m \\ j\end{array}\right)=\frac{(n-m) !}{j !(n-m-j) !}$. One can see that the first two terms in the last equation represent the cumulative distributive function of bivariate binomial distribution. Thus

$$
C=S_{0} B\left(a, b ; p^{\prime} ; n, n-m\right)-(1+r)^{-(n-m)} K_{2} B\left(a, b ; p^{\prime}, p ; n, n-m\right)-(1+r)^{-m} K_{1} \psi(a, m, p)
$$

where $p^{\prime}=\frac{p u}{(1+r)}, a=\left[\frac{\ln \frac{S^{*}}{S_{0}}-m \ln d}{\ln \left(\frac{u}{d}\right)}\right]+1$ and $b=\left[\frac{\ln \frac{K_{2}}{S_{0}}-n \ln d}{\ln \left(\frac{u}{d}\right)}\right]+1$, and $\psi(\cdot, \cdot ; \cdot)$ is the complementary distribution function of bi-variate Binomial distribution. The formula above gives us the CRR premium of the compound European call on European call option.

\section{Convergence of Empirical Means and Volatilities of Log Returns to Actual Means and Volatilities}

Following is the key theorem that we intend to prove in this section.

Theorem 4.1. Assume that we are in the framework of Theorem 3.1. Then following holds:

i) If $S^{*}$ stock price at time $T_{1}$ (i.e. maturity time for compound option) such that the compound option in 
the money then the empirical mean $\widehat{\mu}_{p} m$ and variance of $\widehat{\sigma}_{p}^{2} m$ of log returns $\ln \left(\frac{S^{*}}{S_{0}}\right)$ converges, respectively, to $\mu T_{1}$ and $\sigma^{2} T_{1}$.

ii) Let $T$ be an instant between $T_{1}$ and $T_{2}$ and $S_{T}$ be the stock price at time $T$. Then the empirical mean $\widehat{\mu}_{p}(n-m)$ and variance of $\widehat{\sigma}_{p}^{2}(n-m)$ of log returns $\ln \left(\frac{S_{T}}{S_{0}}\right)$ converges, respectively, to $\mu T_{2}$ and $\sigma^{2} T_{2}$.

Proof. of the compound option given by formula 2.1a. Let $S^{*}$ be the stock price at $T_{1}$ such that. the price of compound option is in the money. So

$$
\begin{aligned}
S^{*} & =S_{0} U^{i} D^{m-i} \\
\ln \left(\frac{S^{*}}{S_{0}}\right) & =\ln (U)^{i}+\ln (D)^{m-i} \\
\ln \left(\frac{S^{*}}{S_{0}}\right) & =i \ln U+(m-i) \ln D=i \ln \left(\frac{U}{D}\right)+m \ln D .
\end{aligned}
$$

Here $i$ is the binomial random variable with mean $\mathbb{E}[i]=m p$ and variance $\operatorname{Var}[i]=m p(1-p)$. As $m \rightarrow \infty$

$$
\mathbb{E}\left[\ln \left(\frac{S^{*}}{S_{0}}\right)\right]=\mathbb{E}\left[i \ln \left(\frac{U}{D}\right)\right]+\mathbb{E}[m \ln D] .
$$

The expectation of constant remains same, So

$$
\begin{aligned}
\mathbb{E}\left[\ln \left(\frac{S^{*}}{S_{0}}\right)\right] & =\mathbb{E}[i]\left[\ln \left(\frac{U}{D}\right)\right]+[m \ln D] \\
& =m p\left[\ln \left(\frac{U}{D}\right)\right]+[m \ln D] \\
& =\left[p \ln \left(\frac{U}{D}\right)+\ln D\right] m .
\end{aligned}
$$

We suppose that the empirical mean value of $\ln \left(\frac{S^{*}}{S_{0}}\right)$ is $\widehat{\mu}_{p} m$ and represent it as:

$$
\widehat{\mu}_{p} m=\left[p \ln \left(\frac{U}{D}\right)+\ln D\right] m
$$

and

$$
\operatorname{Var}\left[\ln \left(\frac{S^{*}}{S_{0}}\right)\right]=\operatorname{Var}\left[i \ln \left(\frac{U}{D}\right)\right]+\operatorname{Var}[m \ln D] .
$$

The variance of $(a X+b)$ is $a^{2} \operatorname{Var} X$, hence

$$
\begin{aligned}
\operatorname{Var}\left[\ln \left(\frac{S^{*}}{S_{0}}\right)\right] & =\left[\ln \left(\frac{U}{D}\right)\right]^{2} \operatorname{Var}[i] \\
& =m p(1-p)\left[\ln \left(\frac{U}{D}\right)\right]^{2}
\end{aligned}
$$

We suppose that the empirical variance value of $\ln \left(\frac{S^{*}}{S_{0}}\right)$ is $\widehat{\sigma}_{p}^{2} m$ and represent it as

$$
\widehat{\sigma}_{p}^{2} m=p(1-p)\left[\ln \left(\frac{U}{D}\right)\right]^{2} m
$$


If $\mu T_{1}=\mathbb{E}\left[\ln \left(\frac{S^{*}}{S_{0}}\right)\right]$ and $\sigma T=\operatorname{Var}\left[\ln \left(\frac{S^{*}}{S_{0}}\right)\right]$ represent the values of actual mean and variance, we want $\widehat{\mu}_{p} m$ converges to $\mu T_{1}$ and $\widehat{\sigma}_{p}^{2} m$ approaches to $\sigma T_{1}$ as $m \rightarrow \infty$ :

$$
\lim _{m \rightarrow \infty} m p \ln \left(\frac{U}{D}\right)+\ln D=\mu T_{1}
$$

and

$$
\lim _{m \rightarrow \infty} m p(1-p)\left[\ln \left(\frac{U}{D}\right)\right]^{2}=\sigma^{2} T_{1}
$$

Also keep in view the following relation between $U$ and $D$,

$$
U=\frac{1}{D}
$$

To have these convergence we need to choose the parameters $U, D$ and $p$, which satisfies last three limiting equations. To do so let solve the system of nonlinear equations (4.4), (4.5) and (4.6), for $U, D$ and $p$. Ignore the limits in the mentioned equations. Using equation (4.6) into (4.4), we may infer that

$$
\begin{aligned}
p \ln \left(U^{2}\right)+\ln \frac{1}{U} & =\frac{\mu T_{1}}{m} \\
\ln U & =\frac{\mu T_{1}}{m(2 p-1)} .
\end{aligned}
$$

Using equation (4.6) into (4.5) we infer,

$$
\begin{aligned}
p(1-p)\left[\ln \left(U^{2}\right)\right]^{2} & =\frac{\sigma T_{1}}{m} \\
{[\ln U]^{2} } & =\frac{\sigma T_{1}}{4 p(1-p) m} .
\end{aligned}
$$

Substituting the equation (4.7) into last equation we get,

$$
\left(\frac{\mu T_{1}}{m(2 p-1)}\right)^{2}=\frac{\sigma T_{1}}{4 p(1-p) m}
$$

On solving the above quadratic equation for $p$ we get,

$$
p=\frac{1}{2}+\frac{1}{2} \frac{\mu}{\sigma} \sqrt{\frac{T_{1}}{m}}
$$

Now substituting the value of $p$ from (4.8) into getting the value of $U$ by using equation (4.7)

$$
\begin{aligned}
\ln U & =\frac{\mu T_{1}}{m} \cdot \frac{1}{\left(2\left(\frac{1}{2}+\frac{1}{2} \frac{\mu}{\sigma} \sqrt{\frac{T_{1}}{m}}\right)-1\right)} \\
\ln U & =\sigma \sqrt{\frac{T_{1}}{m}} \\
U & =e^{\sigma \sqrt{\frac{T_{1}}{m}}}
\end{aligned}
$$


As $D=\frac{1}{U}$, then the value of $D$ is

$$
D=e^{-\sigma \sqrt{\frac{T_{1}}{m}}}
$$

After plugging the calculated values of $U, D$ and $p$ in equations of $\widehat{\mu}_{p} m$ and $\widehat{\sigma}_{p}^{2} m$, we see how these empirical values approaches to the actual values of mean and variance.

$$
\widehat{\mu}_{p} m=\left[p \ln \left(\frac{U}{D}\right)+\ln D\right] m
$$

Note that $\ln \left(\frac{U}{D}\right)=\ln U-\ln D=\sigma \sqrt{\frac{T_{1}}{m}}+\sigma \sqrt{\frac{T_{1}}{m}}=2 \sigma \sqrt{\frac{T_{1}}{m}}$. Now

$$
\begin{aligned}
\widehat{\mu}_{p} m & =\left\{\left[\frac{1}{2}+\frac{1}{2} \frac{\mu}{\sigma} \sqrt{\frac{T_{1}}{m}}\right]\left[2 \sigma \sqrt{\frac{T_{1}}{m}}\right]-\sigma \sqrt{\frac{T_{1}}{m}} m\right\} \\
& =\left[\sigma \sqrt{\frac{T_{1}}{m}}+\mu\left(\frac{T_{1}}{m}\right)-\sigma \sqrt{\frac{T_{1}}{m}}\right] m \\
\lim _{m \rightarrow \infty} \widehat{\mu}_{p} m & =\mu T_{1} .
\end{aligned}
$$

Next

$$
\begin{aligned}
\widehat{\sigma}_{p}^{2} m & =p(1-p)\left[\ln \left(\frac{U}{D}\right)\right]^{2} m \\
& =\left[\frac{1}{2}+\frac{1}{2} \frac{\mu}{\sigma} \sqrt{\frac{T_{1}}{m}}\right]\left[1-\left\{\frac{1}{2}+\frac{1}{2} \frac{\mu}{\sigma} \sqrt{\frac{T_{1}}{m}}\right\}\right]\left[2 \sigma \sqrt{\frac{T_{1}}{m}}\right]^{2} m \\
& =\left(\frac{1}{4}-\frac{1}{4} \frac{\mu^{2}}{\sigma^{2}} \frac{T_{1}}{m}\right) 4 \sigma^{2} T_{1} \\
\lim _{m \rightarrow \infty} \widehat{\sigma}_{p}^{2} m & =\sigma^{2} T_{1} .
\end{aligned}
$$

Now, at the second expiry of underlying stock: $S_{T}=S_{0} U^{j} D^{(n-m)-j}$

$$
\begin{aligned}
& \ln \left(\frac{S_{T}}{S_{0}}\right)=\ln (U)^{j}+(D)^{(n-m)-j} \\
& \ln \left(\frac{S_{T}}{S_{0}}\right)=j \ln U+{ }^{(n-m)-j} \ln D=j \ln \left(\frac{U}{D}\right)+(n-m) \ln D
\end{aligned}
$$

Here $j$ is the binomial random variable with mean $\mathbb{E}[j]=(n-m) p$ and variance $\operatorname{Var}[j]=(n-m)(1-p) p$. As $(n-m) \rightarrow \infty$

$$
\mathbb{E}\left[\ln \left(\frac{S_{T}}{S_{0}}\right)\right]=\mathbb{E}\left[j \ln \left(\frac{U}{D}\right)\right]+\mathbb{E}[(n-m) \ln D]
$$


The expectation of constant remains same, so

$$
\begin{aligned}
\mathbb{E}\left[\ln \left(\frac{S_{T}}{S_{0}}\right)\right] & =\mathbb{E}[j]\left[\ln \left(\frac{U}{D}\right)\right]+[(n-m) \ln D] \\
& =(n-m) p\left[\ln \left(\frac{U}{D}\right)\right]+[(n-m) \ln D] \\
& =\left[p \ln \left(\frac{U}{D}\right)+\ln D\right](n-m) .
\end{aligned}
$$

We suppose that the empirical mean value of $\ln \left(\frac{S_{T}}{S_{0}}\right)$ is $\widehat{\mu}_{p}(n-m)$ and represent it as:

$$
\widehat{\mu}_{p}(n-m)=\left[p \ln \left(\frac{U}{D}\right)+\ln D\right](n-m)
$$

and

$$
\operatorname{Var}\left[\ln \left(\frac{S_{T}}{S_{0}}\right)\right]=\operatorname{Var}\left[j \ln \left(\frac{U}{D}\right)\right]+\operatorname{Var}[(n-m) \ln D] .
$$

Recall the fact that the $\operatorname{Var}(a X+b)=a^{2} \operatorname{Var} X$. By using this,

$$
\begin{aligned}
\operatorname{Var}\left[\ln \left(\frac{S_{T}}{S_{0}}\right)\right] & =\left[\ln \left(\frac{U}{D}\right)\right]^{2} \operatorname{Var}[j] \\
& =(n-m) p(1-p)\left[\ln \left(\frac{U}{D}\right)\right]^{2}
\end{aligned}
$$

We suppose that the empirical variance value of $\ln \left(\frac{S^{*}}{S_{0}}\right)$ is $\widehat{\sigma}_{p}^{2}(n-m)$ and represent it as:

$$
\widehat{\sigma}_{p}^{2}(n-m)=p(1-p)\left[\ln \left(\frac{U}{D}\right)\right]^{2}(n-m) .
$$

If $\mu T_{2}=\mathbb{E}\left[\ln \left(\frac{S_{T}}{S_{0}}\right)\right]$ and $\sigma T_{2}=\operatorname{Var}\left[\ln \left(\frac{S_{T}}{S_{0}}\right)\right]$ represent the values of actual mean and variance, we want $\widehat{\mu}_{p}(n-m)$ approaches to $\mu T_{2}$ and $\widehat{\sigma}_{p}^{2}(n-m)$ approaches to $\sigma T_{2}$ as $(n-m) \rightarrow \infty$ :

$$
p \ln \left(\frac{U}{D}\right)+\ln D \rightarrow \frac{\mu T_{2}}{(n-m)}
$$

and

$$
p(1-p)\left[\ln \left(\frac{U}{D}\right)\right]^{2} \rightarrow \frac{\sigma T_{2}}{(n-m)}
$$

From the last two equations above, we have to calculate the values of parameters $U, D$ and $p$ subject to constraint $U=\frac{1}{D}$ due to the balance in the tree structure in the binomial model. Calculations are shown below:

$$
p=\frac{1}{2}+\frac{1}{2} \frac{\mu}{\sigma} \sqrt{\frac{T_{2}}{(n-m)}} .
$$

Using the relation $U=\frac{1}{D}$ 


$$
\begin{aligned}
p \ln \left(\frac{U}{D}\right)+\ln D & =\frac{\mu T_{2}}{(n-m)} \\
p \ln (U)^{2}+\ln \left(\frac{1}{U}\right) & =\frac{\mu T_{2}}{(n-m)} \\
\ln U & =\frac{\mu T_{2}}{(n-m)} \cdot \frac{1}{(2 p-1)} .
\end{aligned}
$$

Now plugging the value of $p$

$$
\begin{aligned}
& \ln U=\frac{\mu T_{2}}{(n-m)} \cdot \frac{1}{\left(2\left(\frac{1}{2}+\frac{1}{2} \frac{\mu}{\sigma} \sqrt{\frac{T_{2}}{(n-m)}}\right)-1\right)} \\
& \ln U=\frac{\mu T_{2}}{(n-m)} \cdot \frac{1}{\left(\frac{\mu}{\sigma} \sqrt{\frac{T_{2}}{(n-m)}}\right)} \\
& \ln U=\frac{T_{2}}{(n-m)} \cdot \frac{\sigma}{\left(\sqrt{\frac{T_{2}}{(n-m)}}\right)} .
\end{aligned}
$$

After solving the powers

$$
\begin{aligned}
\ln U & =\sigma \sqrt{\frac{T_{2}}{(n-m)}} \text { or } \\
U & =e^{\sigma \sqrt{\frac{T_{2}}{(n-m)}}}
\end{aligned}
$$

As $D=\frac{1}{U}$, then the value of $D$ is

$$
D=e^{-\sigma \sqrt{\frac{T_{2}}{(n-m)}}}
$$

After plugging the calculated values of $U, D$ and $p$ in the $\widehat{\mu}_{p}(n-m)$ and $\widehat{\sigma}_{p}^{2}(n-m)$, we see how these empirical values approaches to the actual values of mean and variance.

$$
\widehat{\mu}_{p}(n-m)=\left[p \ln \left(\frac{U}{D}\right)+\ln D\right](n-m)
$$

Note that $\ln \left(\frac{U}{D}\right)=\ln U-\ln D=\sigma \sqrt{\frac{T_{2}}{(n-m)}}+\sigma \sqrt{\frac{T_{2}}{(n-m)}}=2 \sigma \sqrt{\frac{T_{2}}{(n-m)}}$. Now,

$$
\begin{aligned}
\widehat{\mu}_{p}(n-m) & =\left[\frac{1}{2}+\frac{1}{2} \frac{\mu}{\sigma} \sqrt{\frac{T_{2}}{(n-m)}}\right]\left[2 \sigma \sqrt{\frac{T_{2}}{(n-m)}}-\sigma \sqrt{\frac{T_{2}}{(n-m)}}\right](n-m) \\
& =\left[\sigma \sqrt{\frac{T_{2}}{(n-m)}}+\mu\left(\frac{T_{2}}{(n-m)}\right)-\sigma \sqrt{\frac{T_{2}}{(n-m)}}\right](n-m) \\
\lim _{m \rightarrow \infty} \widehat{\mu}_{p}(n-m) & =\mu T_{2} .
\end{aligned}
$$


Next

$$
\begin{aligned}
\widehat{\sigma}_{p}^{2}(n-m) & =p(1-p)\left[\ln \left(\frac{U}{D}\right)\right]^{2}(n-m) \\
& =\left[\frac{1}{2}+\frac{1}{2} \frac{\mu}{\sigma} \sqrt{\frac{T_{2}}{(n-m)}}\right]\left[1-\left\{\frac{1}{2}+\frac{1}{2} \frac{\mu}{\sigma} \sqrt{\frac{T_{2}}{(n-m)}}\right\}\right] \times \\
& {\left[2 \sigma \sqrt{\frac{T_{2}}{(n-m)}}\right]^{2}(n-m) } \\
& {\left[\frac{1}{2}+\frac{1}{2} \frac{\mu}{\sigma} \sqrt{\frac{T_{2}}{(n-m)}}\right]\left[\frac{1}{2}-\frac{1}{2} \frac{\mu}{\sigma} \sqrt{\frac{T_{2}}{(n-m)}}\right]\left[4 \sigma^{2} T_{2}\right] } \\
& =\left(\frac{1}{4}-\frac{1}{4} \frac{\mu^{2}}{\sigma^{2}} \frac{T_{2}}{(n-m)}\right) 4 \sigma^{2} T_{2} \\
\lim _{m \rightarrow \infty} \widehat{\sigma}_{p}^{2}(n-m) & =\sigma^{2} T_{2} .
\end{aligned}
$$

\section{Main Convergence Result}

In this section we prove the second most important result that the established general CRR formula for binomial pricing formula of a Compound call option, to the well-known continuous-time Black-Scholes formula for European Compound option. We state this assertion in the form of following Theorem.

Theorem 5.1. Assume that we are in assumptions of Theorem 3.1 and Theorem 4.1. the fair price at time 0 of a European compound call option with expiry $m$ and strike $K_{1}$, contingent upon a European call option with expiry $n$ and strike $K_{2}$, can be given as:

$$
\begin{gathered}
C=S_{0} B\left(a, b ; p^{\prime} ; n, n-m\right)-(1+r)^{-(n-m)} K_{2} B\left(a, b ; p^{\prime}, p ; n, n-m\right) \\
-(1+r)^{-m} K_{1} \psi(a ; m, p),
\end{gathered}
$$

converges in the distribution to the following continuous time price of European compound call option with expiry $T_{1}$ and strike $K_{1}$, contingent upon a European call option with expiry $T_{2}$ and strike $K_{2}$,

$$
S_{0} N\left(d_{1}, d_{2} ; \sqrt{\frac{T_{2}}{T_{1}}}\right)-e^{-r T_{2}} K_{2} N\left(d_{1}^{*}, d_{2}^{*} ; \sqrt{\frac{T_{2}}{T_{1}}}\right)-e^{-r T_{1}} K_{1} N\left(d_{1}^{*}\right),
$$

where $p=\frac{r-u}{u-d}, p^{\prime}=\frac{p u}{(1+r)}, a=\left[\frac{\ln \frac{S^{*}}{S_{0}}-m \ln d}{\ln \left(\frac{u}{d}\right)}\right]+1$ and $b=\left[\frac{\ln \frac{K_{2}}{S_{0}}-n \ln d}{\ln \left(\frac{u}{d}\right)}\right]+1$, and $B(\cdot, \cdot ; \cdot)$ is the complementary distribution function of bi-variate Binomial distribution and

$$
\begin{aligned}
& d_{1}=\frac{\ln \frac{S}{S^{*}}+\left(\ln (1+r)-\frac{1}{2} \sigma^{2}\right) T_{1}}{\sigma \sqrt{T_{1}}}, d_{1}^{*}=d_{1}+\sigma \sqrt{T_{1}} \\
& d_{2}=\frac{\ln \left(\frac{S}{K_{2}}\right)+\left(\ln (1+r)-\frac{1}{2} \sigma^{2}\right) T_{2}}{\sigma \sqrt{T_{2}}}, d_{2}^{*}=d_{2}+\sigma \sqrt{T_{2}} .
\end{aligned}
$$


Proof. From the last section we know that our choice of parameter $(1+r)^{\frac{T_{1}}{m}}$ ensures that $K_{1}(1+r)^{-m}=$ $K e^{-r T_{1}}$ and $K_{2}(1+r)^{-(n-m)}=K e^{-r T_{2}}$. Next, we are going to show that the CRR price of compound option converges to the continuous time Black-Scholes price of the compound option. To do let us begin with the following observation about the CDF of bivariate Binomial random variables $i, j$, where $i$ and $j$ denotes the number of times the price of underlying option and compound option, has went up, respectively.

$$
\begin{gathered}
1-B\left(a, b ; p^{\prime} ; n, n-m\right)=\mathbb{P}(i \leq a-1, j \leq b-1) \\
=\mathbb{P}\left(\frac{i-m p}{\sqrt{m p(1-p)}} \leq \frac{a-1-m p}{\sqrt{m p(1-p)}}, \frac{j-(n-m) p}{\sqrt{(n-m) p(1-p)}} \leq \frac{b-1-(n-m) p}{\sqrt{(n-m) p(1-p)}}\right)
\end{gathered}
$$

where

$$
\begin{gathered}
a=\min \left\{i \in m ; \quad i \geq \frac{\ln \frac{S^{*}}{S_{0} D^{m}}}{\ln \frac{U}{D}}\right\} \in\left[\frac{\ln \frac{S^{*}}{S_{0} D^{m}}}{\ln \frac{U}{D}}, \frac{\ln \frac{K_{1}}{C D^{m}}}{\ln \frac{U}{D}}+1\right) \\
b=\min \left\{j \in(n-m) ; j \geq \frac{\ln \frac{K_{2}}{S D^{(n-m)}}}{\ln \left(\frac{U}{D}\right)}\right\} \in\left[\frac{\ln \frac{K_{2}}{S D^{(n-m)}}}{\ln \left(\frac{U}{D}\right)}, \frac{\ln \frac{K_{2}}{S D^{(n-m)}}}{\ln \left(\frac{U}{D}\right)}+1\right) .
\end{gathered}
$$

For the convergence to the continuous time, mean and variance of continuously compounded rate of returns of stocks with respect to $\mathbb{P}=(p, 1-p)$ can be given as:

$$
\begin{gathered}
\widehat{\mu}_{p} m=\left[p \ln \left(\frac{U}{D}\right)+\ln D\right] m \text { and } \widehat{\sigma}_{p}^{2} m=m p(1-p)\left[\ln \left(\frac{U}{D}\right)\right]^{2} \\
\widehat{\mu}_{p}(n-m)=\left[p \ln \left(\frac{U}{D}\right)+\ln D\right](n-m) \quad \text { and } \widehat{\sigma}_{p}^{2} m=m p(1-p)\left[\ln \left(\frac{U}{D}\right)\right]^{2} .
\end{gathered}
$$

Recall that $i$ represents the number of times the stock price goes up before the expiry of the compound option i.e. between 0 and $m$. Also recall the equation (4.1) i.e. $\ln \left(\frac{S^{*}}{S_{0}}\right)=i \ln \left(\frac{U}{D}\right)+m \ln D$. Deducing the value of $i$ from this equation we may infer that

$$
\begin{aligned}
\frac{i-m p}{\sqrt{m p(1-p)}} & =\frac{\frac{\ln \left(\frac{S_{T}}{S_{0}}\right)-m \ln D}{\ln \left(\frac{U}{D}\right)}-m p}{\sqrt{m p(1-p)}} \\
& =\frac{\ln \left(\frac{S_{T}}{S_{0}}\right)-m\left(\ln D+p \ln \left(\frac{U}{D}\right)\right)}{\ln \left(\frac{U}{D}\right) \sqrt{m p(1-p)}} .
\end{aligned}
$$

Using the equations (5.3) it follows that,

$$
\frac{i-m p}{\sqrt{m p(1-p)}}=\frac{\ln \left(\frac{S_{T}}{S_{0}}\right)-\widehat{\mu}_{p} m}{\widehat{\sigma}_{p} \sqrt{m}} .
$$

Let us fix $\varepsilon \in([0,1])$. Now since $a=\min \left\{i \in m ; \quad i \geq \frac{\ln \frac{S^{*}}{S_{0} D^{m}}}{\ln \frac{U}{D}}\right\} \in\left[\frac{\ln \frac{S^{*}}{S_{0} D^{m}}}{\ln \frac{U}{D}}, \frac{\ln \frac{K_{1}}{C D^{m}}}{\ln \frac{U}{D}}+1\right)$ therefore:

$$
a-1=\frac{\ln \frac{S^{*}}{S_{0} D^{m}}}{\ln \left(\frac{U}{D}\right)}-\varepsilon=\frac{\ln \frac{S^{*}}{S_{0}}-m \ln D-\varepsilon \ln \left(\frac{U}{D}\right)}{\ln \frac{U}{D}}
$$


and therefore,

$$
\begin{aligned}
\frac{a-1-m p}{\sqrt{m p(1-p)}} & =\frac{\ln \frac{S^{*}}{S_{0}}-m \ln D-\varepsilon \ln \frac{U}{D}-m p \ln \left(\frac{U}{D}\right)}{\ln \frac{U}{D} \sqrt{m p(1-p)}} \\
& =\frac{\ln \frac{S^{*}}{S_{0}}-m\left(p \ln \frac{U}{D}-\ln D\right)-\varepsilon \ln \left(\frac{U}{D}\right)}{\ln \frac{U}{D} \sqrt{m p(1-p)}} \\
& =\frac{\ln \frac{S^{*}}{S_{0}}-\widehat{\mu}_{p} m-\varepsilon \ln \left(\frac{U}{D}\right)}{\widehat{\sigma}_{p} \sqrt{m}} .
\end{aligned}
$$

Next, let us turn towards the random variable $j$ over $(n-m)$ time steps, where $j$ is the number of up moves of the stock price in the interval $m$ to $n$.

$$
\begin{aligned}
\frac{j-(n-m) p}{\sqrt{(n-m)(1-p) p}} & =\frac{\frac{\ln \left(\frac{S_{T}}{S_{0}}\right)-(n-m) \ln D}{\ln \left(\frac{U}{D}\right)}-(n-m) p}{\sqrt{(n-m)(1-p) p}} \\
& =\frac{\ln \left(\frac{S_{T}}{S_{0}}\right)-(n-m) \ln D-\ln \left(\frac{U}{D}\right)(n-m) p}{\sqrt{(n-m)(1-p) p}} .
\end{aligned}
$$

Using the equations (5.3) it follows that

$$
\frac{j-(n-m) p}{\sqrt{(n-m)(1-p) p}}=\frac{\ln \left(\frac{S_{T}}{S_{0}}\right)-\widehat{\mu}_{p}(n-m)}{\widehat{\sigma}_{p} \sqrt{N}} .
$$

Again since $b=\min \left\{j \in(n-m) ; j \geq \frac{\ln \frac{K_{2}}{S D^{(n-m)}}}{\ln \left(\frac{U}{D}\right)}\right\} \in\left[\frac{\ln \frac{K_{2}}{S D^{(n-m)}}}{\ln \left(\frac{U}{D}\right)}, \frac{\ln \frac{K_{2}}{S D^{(n-m)}}}{\ln \left(\frac{U}{D}\right)}+1\right)$ so it follows that

$$
b-1=\frac{\ln \frac{K_{2}}{S D^{(n-m)}}}{\ln \frac{U}{D}}-\varepsilon=\frac{\ln \left(\frac{K_{2}}{S}\right)-(n-m) \ln D-\varepsilon \ln \left(\frac{U}{D}\right)}{\ln \frac{U}{D}}
$$

and therefore,

$$
\begin{aligned}
\frac{b-1-(n-m) p}{\sqrt{(n-m)(1-p) p}} & =\frac{\ln \left(\frac{K_{2}}{S}\right)-(n-m) \ln D-\varepsilon \ln \left(\frac{U}{D}\right)-(n-m) p \ln \left(\frac{U}{D}\right)}{\ln \frac{U}{D} \sqrt{(n-m) p(1-p)}} \\
& =\frac{\ln \left(\frac{K_{2}}{S}\right)-(n-m)\left(p \ln \left(\frac{U}{D}\right)-\ln D\right)-\varepsilon \ln \left(\frac{U}{D}\right)}{\ln \frac{U}{D} \sqrt{(n-m) p(1-p)}} \\
& =\frac{\ln \left(\frac{K_{2}}{S}\right)-\widehat{\mu}_{p}(n-m)-\varepsilon \ln \left(\frac{U}{D}\right)}{\widehat{\sigma}_{p} \sqrt{(n-m)}} .
\end{aligned}
$$

Using equations (5.5), (5.6), (5.7) and (5.8) into (5.2), it follows that,

$$
\begin{gathered}
1-B\left(a, b ; p^{\prime} ; n, n-m\right) \\
=\mathbb{P}\left(\begin{array}{c}
\frac{\ln \left(\frac{S_{T}}{S_{0}}\right)-\widehat{\mu}_{p} m}{\widehat{\sigma}_{p} \sqrt{m}} \leq \frac{\ln \frac{S^{*}}{S_{0}}-\widehat{\mu}_{p} m-\varepsilon \ln \left(\frac{U}{D}\right)}{\widehat{\sigma}_{p} \sqrt{m}} \\
\frac{\ln \left(\frac{S_{T}}{S_{0}}\right)-\widehat{\mu}_{p}(n-m)}{\widehat{\sigma}_{p} \sqrt{n-m}} \leq \frac{\ln \left(\frac{K_{2}}{S}\right)-\widehat{\mu}_{p}(n-m)-\varepsilon \ln \left(\frac{U}{D}\right)}{\widehat{\sigma}_{p} \sqrt{(n-m)}}
\end{array}\right) .
\end{gathered}
$$


To proceed further we want to show that $\widehat{\mu}_{p} m \rightarrow\left(\ln (1+r)-\frac{1}{2} \sigma^{2}\right) T_{1}$ and $\widehat{\mu}_{p}(n-m) \rightarrow$ $\left(\ln (1+r)-\frac{1}{2} \sigma^{2}\right) T_{2}$ as $m \rightarrow \infty$ and $n \rightarrow \infty$, respectively. We will prove first the convergence, and the second will follow the analogue argument. Recall that between the time 0 to $m$, the value of $m$ can be given as,

$$
p=\frac{1}{2}+\frac{1}{2} \frac{\mu}{\sigma} \sqrt{\frac{T_{1}}{m}}
$$

In order to show that $\widehat{\mu}_{p} m \rightarrow\left(\ln (1+r)-\frac{1}{2} \sigma^{2}\right) T_{1}$ as $m \rightarrow \infty$, it is sufficient to show that,

$$
\begin{aligned}
p= & \frac{1}{2}+\frac{1}{2} \frac{\mu}{\sigma} \sqrt{\frac{T_{1}}{m}} \rightarrow \frac{1}{2}+\frac{1}{2} \frac{\left(\ln (1+r)-\frac{1}{2} \sigma^{2}\right)}{\sigma} \sqrt{\frac{T_{1}}{m}} \text { as } m \rightarrow \infty \\
& \text { or } 2 \sqrt{m}\left(p-\frac{1}{2}\right) \rightarrow \frac{\left(\ln (1+r)-\frac{1}{2} \sigma^{2}\right)}{\sigma} \sqrt{T_{1}} \text { as } m \rightarrow \infty .
\end{aligned}
$$

Or it is sufficient to show that,

$$
\lim _{m \rightarrow \infty} 2 \sqrt{m}\left(p-\frac{1}{2}\right)=\frac{\left(\ln (1+r)-\frac{1}{2} \sigma^{2}\right)}{\sigma} \sqrt{T_{1}} .
$$

Let us consider the term on left hand side of last equation. Using value of $p$ from

$$
\begin{aligned}
2 \sqrt{m}\left(p-\frac{1}{2}\right) & =2 \sqrt{m}\left(\frac{(1+r)^{\frac{T_{1}}{m}}-D}{U-D}-\frac{1}{2}\right) \\
& =2 \sqrt{m}\left(\frac{2(1+r)^{\frac{T_{1}}{m}}-2 D-U+D}{2(U-D)}\right) \\
& =\sqrt{m}\left(\frac{2 \frac{(1+r)^{\frac{T_{1}}{m}}}{D}-\frac{U}{D}-1}{\frac{U}{D}-1}\right) .
\end{aligned}
$$

On substituting $(1+r)^{\frac{T_{1}}{m}}=e^{\left(\frac{T_{1}}{m}\right) \ln (1+r)}, U=e^{\sigma \sqrt{\frac{T_{1}}{m}}}$ and $D=e^{-\sigma \sqrt{\frac{T_{1}}{m}}}\left(\operatorname{or} \frac{U}{D}=e^{2 \sigma \sqrt{\frac{T_{1}}{m}}}\right)$ into last equation

$$
2 \sqrt{m}\left(p-\frac{1}{2}\right)=\sqrt{m}\left(\frac{2 e^{\left(\frac{T_{1}}{m}\right) \ln (1+r)+\sigma \sqrt{\frac{T_{1}}{m}}}-e^{2 \sigma \sqrt{\frac{T_{1}}{m}}}-1}{e^{2 \sigma \sqrt{\frac{T_{1}}{m}}}-1}\right) .
$$

Taking limit $m \rightarrow \infty$,

$$
\lim _{m \rightarrow \infty} 2 \sqrt{m}\left(p-\frac{1}{2}\right)=\lim _{m \rightarrow \infty} \frac{2 e^{\left(\frac{T_{1}}{m}\right) \ln (1+r)+\sigma \sqrt{\frac{T_{1}}{m}}}-e^{2 \sigma \sqrt{\frac{T_{1}}{m}}}-1}{\left(e^{2 \sigma \sqrt{\frac{T_{1}}{m}}}-1\right) m^{-\frac{1}{2}}},\left(\frac{0}{0} \text { form }\right) .
$$

Using L'Hospital rule we may infer that, $\lim _{m \rightarrow \infty} 2 \sqrt{m}\left(p-\frac{1}{2}\right)=$

$$
\begin{aligned}
& =\lim _{m \rightarrow \infty} \frac{2 e^{\left(\frac{T_{1}}{m}\right) \ln (1+r)+\sigma \sqrt{\frac{T_{1}}{m}}}-e^{2 \sigma \sqrt{\frac{T_{1}}{m}}}-1}{\left(e^{2 \sigma \sqrt{\frac{T_{1}}{m}}}-1\right) m^{-\frac{1}{2}}} \\
& =\lim _{m \rightarrow \infty} \frac{2 e^{\left(\frac{T_{1}}{m}\right) \ln (1+r)+\sigma \sqrt{\frac{T_{1}}{m}}}\left(m^{-2} T_{1} \ln (1+r)+\frac{1}{2} m^{-\frac{3}{2}} \sigma \sqrt{T_{1}}\right)-e^{2 \sigma \sqrt{\frac{T_{1}}{m}}} m^{-\frac{3}{2}} \sigma \sqrt{T_{1}}}{e^{2 \sigma \sqrt{\frac{T_{1}}{m}}}\left(m^{-2} T_{1} \ln (1+r)+\frac{1}{2} m^{-\frac{3}{2}}\right)-\frac{1}{2} m^{-\frac{3}{2}}} .
\end{aligned}
$$


On dividing the numerator and the denominator by $m^{-\frac{3}{2}} e^{2 \sigma \sqrt{\frac{T_{1}}{m}}}$, we get

$$
=\lim _{m \rightarrow \infty} \frac{2 e^{\left(\frac{T_{1}}{m}\right) \ln (1+r)+\sigma \sqrt{\frac{T_{1}}{m}}}\left(m^{-\frac{1}{2}} T_{1} \ln (1+r)+\frac{1}{2} \sigma \sqrt{T_{1}}\right)-\sigma \sqrt{T_{1}}}{\sigma \sqrt{\frac{T_{1}}{m}}+\frac{1}{2}-\frac{1}{2} e^{2 \sigma \sqrt{\frac{T_{1}}{m}}}},\left(\frac{0}{0} \text { form }\right) .
$$

Again using L'Hospital rule, we infer,

$$
=\lim _{m \rightarrow \infty} \frac{2 e^{\left(\frac{T_{1}}{m}\right) \ln (1+r)+\sigma \sqrt{\frac{T_{1}}{m}}}\left(-m^{-2} T_{1} \ln (1+r)+\frac{1}{2} m^{-\frac{3}{2}} \sigma \sqrt{T_{1}}\right)\left(m^{-\frac{1}{2}} T_{1} \ln (1+r)+\frac{1}{2} \sigma \sqrt{T_{1}}\right)}{-\frac{1}{2} m^{-\frac{3}{2}} T_{1} \ln (1+r)} .
$$

On multiplication of $2 m^{\frac{3}{2}}$ into the numerator and denominator and simplifying it follows that,

$$
\begin{gathered}
=\lim _{m \rightarrow \infty} \frac{4 e^{\left(\frac{T_{1}}{m}\right) \ln (1+r)+\sigma \sqrt{\frac{T_{1}}{m}}}\left(m^{-1}\left(T_{1} \ln (1+r)\right)^{2}-m^{\frac{1}{2}} T_{1}^{\frac{3}{2}} \sigma \ln (1+r)+\frac{1}{4} T_{1} \sigma-\frac{1}{2} T_{1} \ln (1+r)\right)}{-\sigma \sqrt{T_{1}}\left(1+e^{-2 \sigma \sqrt{\frac{T_{1}}{m}}}\right)} \\
=\frac{4\left(\frac{1}{4} \sigma^{2}-\frac{1}{2} T_{1} \ln (1+r)\right)}{-2 \sigma \sqrt{T_{1}}} \\
\lim _{m \rightarrow \infty} 2 \sqrt{m}\left(p-\frac{1}{2}\right)=\frac{\ln (1+r)-\frac{\sigma^{2}}{2}}{\sigma} \sqrt{T_{1}} .
\end{gathered}
$$

On returning the value of $p$,

$$
\lim _{m \rightarrow \infty} p=\lim _{m \rightarrow \infty} \frac{1}{2}+\frac{1}{2} \frac{\mu}{\sigma} \sqrt{\frac{T_{1}}{m}}=\frac{1}{2}+\frac{1}{2} \frac{\left(\ln (1+r)-\frac{1}{2} \sigma^{2}\right)}{\sigma} \sqrt{\frac{T_{1}}{m}} .
$$

On the same lines we can prove that, between $m$ and $n$,

$$
\lim _{m \rightarrow \infty} \frac{1}{2}+\frac{1}{2} \frac{\mu}{\sigma} \sqrt{\frac{T_{2}}{n-m}}=\frac{1}{2}+\frac{1}{2} \frac{\left(\ln (1+r)-\frac{1}{2} \sigma^{2}\right)}{\sigma} \sqrt{\frac{T_{2}}{n-m}} .
$$

Now recall from the equation (5.3),

$$
\widehat{\mu}_{p} m=\left[p \ln \left(\frac{U}{D}\right)+\ln D\right] m
$$

Substitute the values of $U=e^{\sigma \sqrt{\frac{T_{1}}{m}}}$ and $D=e^{-\sigma \sqrt{\frac{T_{1}}{m}}}\left(\operatorname{or} \frac{U}{D}=e^{2 \sigma \sqrt{\frac{T_{1}}{m}}}\right)$, we get,

$$
\begin{aligned}
\widehat{\mu}_{p} m & =\left[p \ln \left(\frac{U}{D}\right)+\ln D\right] m \\
\widehat{\mu}_{p} m & =\left[p \ln \left(e^{2 \sigma \sqrt{\frac{T_{1}}{m}}}\right)+\ln e^{-\sigma \sqrt{\frac{T_{1}}{m}}}\right] m \\
& =\left[2 \sigma p \sqrt{\frac{T_{1}}{m}}-\sigma \sqrt{\frac{T_{1}}{m}}\right] m \\
& =[2 p-1] m \sigma \sqrt{\frac{T_{1}}{m}} .
\end{aligned}
$$


Taking limit $m \rightarrow \infty$ and using equation (5.10), it follows that,

$$
\begin{aligned}
\lim _{m \rightarrow \infty} \widehat{\mu}_{p} m & =\lim _{m \rightarrow \infty}\left[2\left(\frac{1}{2}+\frac{1}{2} \frac{\left(\ln (1+r)-\frac{1}{2} \sigma^{2}\right)}{\sigma} \sqrt{\frac{T_{1}}{m}}\right)-1\right] m \sigma \sqrt{\frac{T_{1}}{m}} \\
& =\lim _{m \rightarrow \infty}\left[\frac{\left(\ln (1+r)-\frac{1}{2} \sigma^{2}\right)}{\sigma} \sqrt{\frac{T_{1}}{m}}\right] m \sigma \sqrt{\frac{T_{1}}{m}} \\
\lim _{m \rightarrow \infty} \widehat{\mu}_{p} m & =\left(\ln (1+r)-\frac{1}{2} \sigma^{2}\right) T_{1} .
\end{aligned}
$$

Now we turn towards the next interval i.e. $m$ to $n$. Recall the equation (5.4),

$$
\widehat{\mu}_{p}(n-m)=\left[p \ln \left(\frac{U}{D}\right)+\ln D\right](n-m)
$$

Substitute the values of $U=e^{\sigma \sqrt{\frac{T_{2}}{n-m}}}$ and $D=e^{-\sigma \sqrt{\frac{T_{2}}{n-m}}}\left(\operatorname{or} \frac{U}{D}=e^{2 \sigma \sqrt{\frac{T_{2}}{n-m}}}\right.$ ), we get,

$$
\begin{aligned}
\widehat{\mu}_{p}(n-m) & =\left[p \ln \left(e^{2 \sigma \sqrt{\frac{T_{2}}{n-m}}}\right)+\ln e^{-\sigma \sqrt{\frac{T_{2}}{n-m}}}\right](n-m) \\
& =\left[2 \sigma p \sqrt{\frac{T_{2}}{n-m}}-\sigma \sqrt{\frac{T_{2}}{n-m}}\right](n-m) \\
& =[2 p-1](n-m) \sigma \sqrt{\frac{T_{2}}{n-m}} .
\end{aligned}
$$

Taking limit $(n-m) \rightarrow \infty$ and using equation (5.11), it follows that,

$$
\begin{aligned}
\lim _{(n-m) \rightarrow \infty} \widehat{\mu}_{p}(n-m) & =\left[2\left(\frac{1}{2}+\frac{1}{2} \frac{\left(\ln (1+r)-\frac{1}{2} \sigma^{2}\right)}{\sigma} \sqrt{\frac{T_{2}}{n-m}}\right)-1\right](n-m) \sigma \sqrt{\frac{T_{2}}{n-m}} \\
& =\lim _{m \rightarrow \infty}\left[\frac{\left(\ln (1+r)-\frac{1}{2} \sigma^{2}\right)}{\sigma} \sqrt{\frac{T_{2}}{n-m}}\right](n-m) \sigma \sqrt{\frac{T_{2}}{n-m}} \\
\lim _{(n-m) \rightarrow \infty} \widehat{\mu}_{p}(n-m) & =\left(\ln (1+r)-\frac{1}{2} \sigma^{2}\right) T_{2} .
\end{aligned}
$$

Now let us return to the equation (5.6). Take limit $m \rightarrow \infty$ and $\varepsilon \rightarrow 0$, and using the equations (5.12) and (4.11), we consider the following limit.

$$
\lim _{m \rightarrow \infty} \lim _{\varepsilon \rightarrow 0} \frac{\ln \frac{S^{*}}{S_{0}}-\widehat{\mu}_{p} m-\varepsilon \ln \left(\frac{U}{D}\right)}{\widehat{\sigma}_{p} \sqrt{m}}=\frac{\ln \frac{S^{*}}{S_{0}}-\left(\ln (1+r)-\frac{1}{2} \sigma^{2}\right) T_{1}}{\sigma \sqrt{T_{1}}}=d_{1} .
$$

Similarly consider the equation (5.8). By application of limit $(n-m) \rightarrow \infty$ and $\varepsilon \rightarrow 0$, and using the equation (4.14) and (5.13), we consider the following limit,

$$
\lim _{m \rightarrow \infty} \lim _{\varepsilon \rightarrow 0} \frac{\ln \left(\frac{K_{2}}{S}\right)-\widehat{\mu}_{p}(n-m)-\varepsilon \ln \left(\frac{U}{D}\right)}{\widehat{\sigma}_{p} \sqrt{(n-m)}}=\frac{\ln \left(\frac{K_{2}}{S}\right)-\left(\ln (1+r)-\frac{1}{2} \sigma^{2}\right) T_{2}}{\sigma \sqrt{T_{2}}}=d_{2} .
$$


Thus we are in a position to claim our key convergence, using bivariate Binomial convergence to bivariate binomial from [16] (page 9) and Theorem 4.1 we infer that

$$
\begin{aligned}
1-B\left(a, b ; p^{\prime} ; n, n-m\right) & =\mathbb{P}\left(\begin{array}{c}
\frac{\ln \left(\frac{S_{T}}{S_{0}}\right)-\widehat{\mu}_{p} m}{\widehat{\sigma}_{p} \sqrt{m}} \leq \frac{\ln \frac{S^{*}}{S_{0}}-\widehat{\mu}_{p} m-\varepsilon \ln \left(\frac{U}{D}\right)}{\widehat{\sigma}_{p} \sqrt{m}}, \\
\frac{\ln \left(\frac{S_{T}}{S_{0}}\right)-\widehat{\mu}_{p}(n-m)}{\widehat{\sigma}_{p} \sqrt{N}} \leq \frac{\ln \left(\frac{K_{2}}{S}\right)-\widehat{\mu}_{p}(n-m)-\varepsilon \ln \left(\frac{U}{D}\right)}{\widehat{\sigma}_{p} \sqrt{(n-m)}}
\end{array}\right) \\
& \rightarrow N\left(-d_{1},-d_{2} ; \sqrt{\frac{T_{2}}{T_{1}}}\right)
\end{aligned}
$$

as $(n-m), m \rightarrow \infty$, where

$$
\begin{gathered}
\frac{\ln \frac{S^{*}}{S_{0}}-\left(\ln (1+r)-\frac{1}{2} \sigma^{2}\right) T_{1}}{\sigma \sqrt{T_{1}}}:=-d_{1} \\
\frac{\ln \left(\frac{K_{2}}{S}\right)-\left(\ln (1+r)-\frac{1}{2} \sigma^{2}\right) T_{2}}{\sigma \sqrt{T_{2}}}:=-d_{2} .
\end{gathered}
$$

Since $1-N\left(-d_{1},-d_{2} ; \sqrt{\frac{T_{2}}{T_{1}}}\right)=N\left(d_{1}, d_{2}\right)$, hence

$$
B\left(a, b ; p^{\prime} ; n, n-m\right) \rightarrow N\left(d_{1}, d_{2} ; \sqrt{\frac{T_{2}}{T_{1}}}\right) .
$$

where,

$$
\begin{gathered}
\frac{\ln \frac{S}{S^{*}}+\left(\ln (1+r)-\frac{1}{2} \sigma^{2}\right) T_{1}}{\sigma \sqrt{T_{1}}}:=d_{1} \\
\frac{\ln \left(\frac{S}{K_{2}}\right)+\left(\ln (1+r)-\frac{1}{2} \sigma^{2}\right) T_{2}}{\sigma \sqrt{T_{2}}}:=d_{2} .
\end{gathered}
$$

Precisely on the same line of argument we can show that

$$
B\left(a, b ; p, p^{\prime} ; n, n-m\right) \rightarrow N\left(d_{1}^{*}, d_{2}^{*} ; \sqrt{\frac{T_{2}}{T_{1}}}\right)
$$

where,

$$
\begin{aligned}
& d_{1}^{*}=d_{1}+\sigma \sqrt{T_{1}} \\
& d_{2}^{*}=d_{2}+\sigma \sqrt{T_{2}}
\end{aligned}
$$

and $N\left(\cdot, \cdot ; \sqrt{\frac{T_{2}}{T_{1}}}\right)$ is the bivariate standard normal CDF with correlation coefficient $\sqrt{\frac{T_{2}}{T_{1}}}$. Finally we will deal with the one-dimensional convergence i.e. $\psi(i \geq a, m, p) \rightarrow N\left(d_{1}^{*}\right)$ as $m \rightarrow \infty$. Again

$$
\begin{aligned}
1-\psi(i \geq a, m, p) & =\mathbb{P}(i \leq a-1) \\
& =\mathbb{P}\left(\frac{i-m p}{\sqrt{m p(1-p)}} \leq \frac{a-1-m p}{\sqrt{m p(1-p)}}\right)
\end{aligned}
$$

where

$$
a=\min \left\{i \in m ; \quad i \geq \frac{\ln \frac{S^{*}}{S_{0} D^{m}}}{\ln \frac{U}{D}}\right\} \in\left[\frac{\ln \frac{S^{*}}{S_{0} D^{m}}}{\ln \frac{U}{D}}, \frac{\ln \frac{K_{1}}{C D^{m}}}{\ln \frac{U}{D}}+1\right)
$$


Using equation (5.5) and equation (5.6) into the last equation we infer that

$$
1-\psi(i \geq a, m, p)=\mathbb{P}\left(\frac{\ln \left(\frac{S_{T}}{S_{0}}\right)-\widehat{\mu}_{p} m}{\widehat{\sigma}_{p} \sqrt{m}} \leq \frac{\ln \frac{S^{*}}{S_{0}}-\widehat{\mu}_{p} m-\varepsilon \ln \left(\frac{U}{D}\right)}{\widehat{\sigma}_{p} \sqrt{m}}\right) .
$$

Using the convergence (5.14) and the one dimensional Central Limit Theorem, and arguing in same manner as we argued in the end of the section 4 , we may infer that

$$
\begin{aligned}
1-\psi(i \geq a, m, p) & =\mathbb{P}\left(\frac{\ln \left(\frac{S_{T}}{S_{0}}\right)-\widehat{\mu}_{p} m}{\widehat{\sigma}_{p} \sqrt{m}} \leq \frac{\ln \frac{S^{*}}{S_{0}}-\widehat{\mu}_{p} m-\varepsilon \ln \left(\frac{U}{D}\right)}{\widehat{\sigma}_{p} \sqrt{m}}\right) \\
& \rightarrow N\left(\frac{\ln \frac{S^{*}}{S_{0}}-\left(\ln (1+r)-\frac{1}{2} \sigma^{2}\right) T_{1}}{\sigma \sqrt{T_{1}}}-\sigma \sqrt{T_{1}}\right)
\end{aligned}
$$

as $m \rightarrow \infty$, where $N(\cdot)$ is the c.d.f of standard normal distribution. By the use of symmetry property of the standard normal c.d.f i.e. $1-N(z)=N(-z)$, it follows that,

$$
\psi(i \geq a, m, p)=N\left(\frac{\ln \frac{S}{S^{*}}+\left(\ln (1+r)-\frac{1}{2} \sigma^{2}\right) T_{1}}{\sigma \sqrt{T_{1}}}+\sigma \sqrt{T_{1}}\right)=N\left(d_{1}^{*}\right) .
$$

Hence, we are done with the conclusion that

$$
\begin{aligned}
C= & S_{0} B\left(i \geq a, j \geq b ; p^{\prime} ; n, n-m\right)-(1+r)^{-(n-m)} K_{2} B\left(i \geq a, j \geq b ; p^{\prime}, p ; n, n-m\right) \\
& -(1+r)^{-m} K_{1} \psi(i \geq a, m, p) \\
\rightarrow & S_{0} N\left(d_{1}, d_{2} ; \sqrt{\frac{T_{2}}{T_{1}}}\right)-e^{-r T_{2}} K_{2} N\left(d_{1}^{*}, d_{2}^{*} ; \sqrt{\frac{T_{2}}{T_{1}}}\right)-e^{-r T_{1}} K_{1} N\left(d_{1}^{*}\right),
\end{aligned}
$$

where

$$
\begin{aligned}
& d_{1}=\frac{\ln \frac{S}{S^{*}}+\left(\ln (1+r)-\frac{1}{2} \sigma^{2}\right) T_{1}}{\sigma \sqrt{T_{1}}}, d_{1}^{*}=d_{1}+\sigma \sqrt{T_{1}} \\
& d_{2}=\frac{\ln \left(\frac{S}{K_{2}}\right)+\left(\ln (1+r)-\frac{1}{2} \sigma^{2}\right) T_{2}}{\sigma \sqrt{T_{2}}}, d_{2}^{*}=d_{2}+\sigma \sqrt{T_{2}}
\end{aligned}
$$

Thus we have shown that our developed CRR formula converges to the standard well-known continuous time Black-Scholes price of the compound option.

\section{Conclusion}

The paper provides a comprehensive treatment of the binomial pricing of option of financial derivatives, in general, and options in particular. Following two new results have been proven.

1. The Cox-Ross Rubinstein(CRR) type formula has been derived for risk-neutral pricing of the European style Compound call option on European call option.

2. It has been shown explicitly that for a suitable choice of parameters, the CRR formula to price the compound call option of on call, converges to the well known, continuous time version of the 
Black-Scholes price of the same option. Simulation of our derived formula will be helpful for financial market.

Conflicts of Interest: The author(s) declare that there are no conflicts of interest regarding the publication of this paper.

\section{REFERENCES}

[1] Agliardi, E., Agliardi, R., A closed-form solution for multi-compound options. Risk Lett. 1 (2) (2004), 12.

[2] Black, F., \& Scholes, M. The pricing of options and corporate liabilities. J. Politic. Econ. 81 (3) (1973), 637-654.

[3] Cassimon, D., Engelen, P.-J., Thomassen, L., \& Van Wouwe, M. The valuation of a NDA using a 6-fold compound option. Res. Policy, 33 (1) (2004), 41-51.

[4] Chiarella, C., Griebsch, S., Kang, B., A comparative study on time-efficient methods to price compound options in the Heston model. Comput. Math. Appl. 67 (6) (2014), 12541270.

[5] Chiarella, C., Kang, B., The evaluation of American compound option prices under stochastic volatility and stochastic interest rates. J. Comput. Financ. 14 (9) (2011), 121.

[6] Cortazar, G., \& Schwartz, E. S. A compound option model of production and intermediate inventories. J. Business, 66 (4) (1993), 517-540.

[7] Cutland, N. J., \& Roux, A. Derivative pricing in discrete time, Springer Science \& Business Media, 2012.

[8] Cox, J. C., \& Ross, S. A. The valuation of options for alternative stochastic processes. J. Financ. Econ. 3 (1-2) (1976), 145-166.

[9] Geske, R. The valuation of compound options. J. Financ. Econ. 7 (1) (1979), 63-81.

[10] Geske, R. The valuation of corporate liabilities as compound options. J. Financ. Quant. Anal. 12 (4) (1977), $541-552$.

[11] Griebsch, S.A. The evaluation of European compound option prices under stochastic volatility using Fourier transform techniques. Rev. Deriv. Res. 16 (2) (2013), 135165.

[12] Gukhal, C.R. The compound option approach to American options on jump diffusion. J. Econ. Dyn. Control 28 (10) (2004), 20552074 .

[13] Fouque, J.-P., Han, C.-H. Evaluation of compound options using perturbation approximation. J. Comput. Financ. 9 (1) (2005), 4161.

[14] Hull, J. C. Options, futures, and other derivatives: Pearson Education India, 2006.

[15] Lajeri-Chaherli, F., 2002. A note on the valuation of compound options. J. Futures Markets, 22 (11), 11031115.

[16] Marshall, A. W., and I. Olkin, A Family of Bivariate Distributions Generated by the Bivariate Bernoulli Distribution, J. Amer. Stat. Assoc. 80 (1985), 332-338.

[17] Merton, R. C. Option pricing when underlying stock returns are discontinuous. J. Financ. Econ. 3 (1-2) (1976), $125-144$.

[18] Samuelson P.A. Rational Theory of Warrant Pricing. In: Grnbaum F., van Moerbeke P., Moll V. (eds) Henry P. McKean Jr. Selecta. Contemporary Mathematicians. Birkhuser, Cham. 2015.

[19] Z. Brzeźniak and T. Zastawniak, Basic Stochastic Process. Springer, 1999. 